Caracterización de la gerencia publicitaria y su influencia en el consumidor del sector comercial de la Provincia de Tungurahua

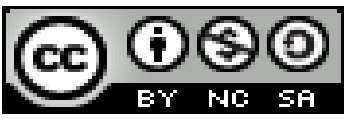

Ciencias económicas y empresariales

Artículo de investigación

\title{
Caracterización de la gerencia publicitaria y su influencia en el consumidor del sector comercial de la Provincia de Tungurahua
}

\section{Characterization of advertising management and its influence on the consumer of the commercial sector of the Province of Tungurahua}

\section{Caracterização da gestão publicitária e sua influência sobre o consumidor do setor comercial da Província de Tungurahua}

Carlos V. Mejía-Vayas ${ }^{\mathrm{I}}$

carlosvmejia@uta.edu.ec

María C. Abril-Freire ${ }^{\text {II }}$

mariacabril@uta.edu.ec
Leonardo G. Ballesteros-López ${ }^{\mathrm{III}}$ leonardogballesteros@uta.edu.ec

César A. Guerrero-Velástegui IV ca.guerrero@uta.edu.ec

Deysi C. Tuala-Andachi ${ }^{\mathrm{V}}$

deysi_20.93@hotmail.com

Recibido: 14 de julio de 2018 * Corregido: 05 de agosto de 2018 * Aceptado: 10 de septiembre de 2018

I. Magister en Tecnología de la Información y Multimedia Educativa, Magister Administración, Ingeniero en Empresas, Ingeniero de Sistemas y Computación, Facultad de Ciencias Administrativas, Universidad Técnica de Ambato, Ambato, Ecuador.

II. Magister en Administración de Empresas Mención Planeación, Magister en Gestión del Talento Humano, Psicóloga Clínica, Psicóloga Industrial, Facultad de Ciencias Administrativas, Universidad Técnica de Ambato, Ambato, Ecuador.

III. Magister en Gestión Estratégica Empresarial MBA, Ingeniero Comercial, Licenciado en Ciencias Administrativas Administrador en Mercadotecnia, Facultad de Ciencias Administrativas, Universidad Técnica de Ambato, Ambato, Ecuador.

IV. Magister en Administración de Empresas Mención Planeación, Ingeniero de Empresas, Abogado de los Tribunales y Juzgados de la República del Ecuador, Facultad de Ciencias Administrativas, Universidad Técnica de Ambato, Ambato, Ecuador.

v. Estudiante Facultad de Ciencias Administrativas, Universidad Técnica de Ambato, Ambato, Ecuador. 


\section{Resumen}

La presente investigación caracteriza la gerencia publicitaria y analiza la influencia que esta causa en el consumidor del sector comercial de la Provincia de Tungurahua. El estudio tuvo un carácter descriptivo y correlacional que se apoyó en la investigación de campo para aplicar la técnica de la encuesta, con lo que se pudo conocer que la publicidad influye notoriamente en el consumidor y lo cual conlleva a un incremento sustancial en las ventas. La calidad, precio y promociones son los principales factores de publicidad que influyen en el consumidor. Además, que las empresas comerciales se apoyan en medios publicitarios tradicionales como los anuncios televisivos, pero también en medios digitales principalmente en redes sociales como Facebook debido a su facilidad de uso y bajos costos. Razón por la cual se propuso una estrategia sobre publicidad a base de redes sociales en la que se brinda recomendaciones sobre creación, manejo, mantenimiento de estas plataformas y acciones para mejorar las ventas del sector comercial, pues actualmente las redes sociales se han consolidado como un poderoso medio para atender y gestionar clientes mucho más efectivos que cualquier otro tipo de medio.

Palabras clave: publicidad; publicidad; digital, influencia; consumidor; estrategias.

\section{Abstract}

The present investigation characterizes the advertising management and analyzes the influence that this causes in the consumer of the commercial sector of the Province of Tungurahua. The study had a descriptive and correlational character that was based on field research to apply the technique of the survey, with which it was possible to know that advertising has a noticeable influence on the consumer and which leads to a substantial increase in sales. The quality, price and promotions are the main advertising factors that influence the consumer. In addition, commercial companies rely on traditional advertising media such as television ads, but also in digital media mainly on social networks such as Facebook due to its ease of use and low costs. Reason for which a strategy on advertising based on social networks was proposed, which provides recommendations on creation, management, maintenance of these platforms and actions to improve sales in the commercial sector, since social networks have now been consolidated as a powerful means to attend and manage clients much more effective than any other type of medium.

Key words: advertising; advertising; digital, influence; consumer; strategies. 


\section{Resumo}

A presente investigação caracteriza a gestão publicitária e analisa a influência que isso provoca no consumidor do setor comercial da Província de Tungurahua. O estudo teve caráter descritivo e correlacional que foi baseado em pesquisa de campo para aplicação da técnica da pesquisa, com a qual foi possível saber que a publicidade tem uma influência perceptível no consumidor e que leva a um aumento substancial nas vendas. A qualidade, preço e promoções são os principais fatores publicitários que influenciam o consumidor. Além disso, as empresas comerciais contam com meios de publicidade tradicionais, como anúncios de televisão, mas também em mídias digitais, principalmente em redes sociais como o Facebook, devido à facilidade de uso e aos baixos custos. Motivo para o qual foi proposta uma estratégia de publicidade baseada em redes sociais, que fornece recomendações sobre criação, gerenciamento, manutenção dessas plataformas e ações para melhorar as vendas no setor comercial, já que as redes sociais já estão consolidadas como um todo. Meios poderosos para atender e gerenciar clientes muito mais eficazes do que qualquer outro tipo de mídia.

Palavras chave: publicidade; publicidade; digital, influência; consumidor estratégias.

\section{Introducción}

En el contexto de la globalización de mercados y desequilibrios entre oferta y demanda, las empresas buscan cada vez más explorar y llegar a nuevos mercados para promocionar sus productos" Corbacho (2009), en tal sentido la publicidad sigue siendo un instrumento de comunicación de valor inestimable para las empresas, razón por la cual en cada época, la economía y la sociedad diferencian la forma de comunicarse y los soportes que se utilizan para informar y persuadir e influenciar al público objetivo (Barcena, 2014).

Las empresas ecuatorianas de cualquier actividad económica buscan generar un incremento considerable en sus ventas por medio del desarrollo práctico de estrategias que permitan alcanzar una mayor participación en el mercado. Cañar \& Guzmán (2014) mencionan que el manejo de la publicidad en los distintos medios del país hace algunos años atrás estaba dirigido por el sector privado, sin embargo, esto se reformó con la nueva Ley de Comunicación, lo que permitió el fortalecimiento de la producción nacional de los distintos espacios publicitarios. 
Tungurahua es una provincia que ha tenido gran desarrollo en su sector productivo, los más evidentes son el gran movimiento comercial, la presencia de la construcción, textil y la agricultura (Yánez, 2012), debido a que recibe la visita de propios y turistas nacionales e internacionales, razón por la cual existe gran competitividad en todos los sectores comerciales, es por ello que para sobresalir, las empresas deben enfocarse en intensificar sus programas publicitarios, teniendo en consideración que el mercado actual se ha diversificado enormemente, y lo importante de hoy es desarrollar estrategias que se adapten a la realidad e influyan en el consumidor y además funcionen sin importar los cambios internos o externos que se generen, con la finalidad de fidelizar a los clientes y de esa forma lograr su desarrollo empresarial (Cadena, 2014).

En un entorno globalizado y en constante cambio como en el que se encuentra el sector comercial de la provincia de Tungurahua, la capacidad competitiva de las empresas se vuelve un factor crítico a la hora de enfrentar con éxito la competencia, tanto nacional como internacional. Sin embargo, se tiene como denominador común la inadecuada difusión comercial de las empresas, debido a que no se da lugar a un análisis de estrategias de publicidad que les ayude al crecimiento en el mercado local y nacional, generando un declive a corto o largo plazo que los llevará al decrecimiento de la empresa o en el peor de los casos al cierre de las mismas (Oller, 2016).

Para un mayor nivel de comprensión sobre el problema de estudio, a continuación, se exponen los siguientes términos:

\section{Desarrollo}

\section{La publicidad}

Para Esteinou (2014) la publicidad es una actividad comunicativo-cultural que en diferentes propiedades ha sido utilizada en todos los tiempos desde que existe el comercio para dar a conocer a la colectividad los productos, servicios que existen en una sociedad para que estos sean aceptados o adquiridos por la mayoría de los consumidores.

La publicidad puede ser definida como toda forma de comunicación realizada por una persona física, pública o privada en el ejercicio de una actividad comercial, industrial, artesanal o profesional con el 
objetivo de promover de manera directa o indirecta la contratación de bienes muebles o inmuebles, derechos y obligaciones (De Durán, 2014, p.4).

La publicidad es importante tanto para la empresa como para los consumidores, pues por una parte a la empresa le sirve como una herramienta de competencia para atraer a los consumidores y por otra, la publicidad es ventajosa para los consumidores al permitirles ser más específicos durante sus compras y a darse cuenta de nuevos negocios, productos y marcas (Terkan, 2014).

\section{Para Yánez (2015) los objetivos de la publicidad son:}

Informar, es decir dar a conocer el producto o servicio al mercado, sus usos, características, necesidad que satisface y como lo hace. Para lograrlo recurre no sólo a la imagen, sino también al texto.

Persuadir, demostrando que el producto/servicio satisface su necesidad, con el propósito de convencer resultan válidos todo tipo de argumentos, dentro de algunos parámetros sociales.

Recordar, volver a recordarle al consumidor que el producto/servicio está ahí, aunque el producto no cuente con un gran posicionamiento.

\section{Medios publicitarios}

De manera puntual Escribano, Alcaraz, y Fuentes (2014), afirman que los medios publicitarios "son canales de comunicación que se encargan de transmitir los mensajes publicitarios” (p. 287).

La importancia de los medios de comunicación en la publicidad, radica en que son el medio por el cual se anuncian los bienes y servicios que comercializa una empresa, es decir, en base al medio las empresas diseñan los mensajes para atraer a consumidores potenciales. Cabe destacar que en la actualidad se habla sobre los medios tradicionales y no tradicionales. Como medios tradicionales se mencionan a la televisión, radio, prensa escrita y dependiendo el desarrollo de cada país se incluye en la lista también al internet. Por otra parte, como medios no convencionales se entiende a todos los medios distintos a los mencionados anteriormente. 


\section{Influencia de la publicidad}

Desde el punto de vista del Marketing, los mensajes de publicidad persuaden al consumidor generando motivaciones, la publicidad influye en la compra en la realización de las compras diarias o esporádicas de los consumidores, hasta tal punto que si la publicidad no mostrase determinadas marcas o productos estos no podrían llegar a venderse (Núñez, Olarte \& Reinares, 2014).

De acuerdo a Novo (2016) en su trabajo "Publicidad, persuasión y comportamiento del consumidor" reporta que la motivación de compra está influenciada por la publicidad, persuasión y el valor que el consumidor da las marcas, las relaciones que se analizó en el proyecto concluyen que los consumidores son incentivados hacia la compra por medio de distintos mecanismos asociados a los mensajes publicitarios y al deseo de integrarse a "grupos especiales exclusivos" por medio de la utilización de marcas, como signos de distinción de pertenencia al grupo.

\section{Importancia de la publicidad}

La importancia de la publicidad para los negocios radica en que es crucial para el lanzamiento o anuncio de bienes o servicios, promueve bienes, servicios, ideas y eventos, contribuye al aumento de ventas, maximiza el beneficio de un anunciante, crea conciencia en el consumidor, pero requiere de creatividad y uso de la persuasión para obtener resultados además que exige monitoreo de demandaoferta para construir la imagen de una marca (Akrani, 2013).

A más de ello la publicidad es importante tanto para la empresa como para los consumidores, pues por una parte a la empresa le sirve como una herramienta de competencia para atraer a los consumidores y por otra, la publicidad es ventajosa para los consumidores al permitirles ser más específicos durante sus compras y a darse cuenta de nuevos negocios, productos y marcas (Terkan, 2014).

La publicidad se constituye como una actividad con importantes repercusiones económicas y sociales, como instrumento económico la publicidad contribuye en cierta medida a la expansión de ventas, desde una perspectiva social la publicidad tiene gran utilidad por su contenido informativo que beneficia al consumidor en particular y ala la sociedad en general dando a conocer formas para satisfacer necesidades y deseos (Paz, 2014). La publicidad de este modo ha dejado de desempeñar en 
la práctica el papel meramente auxiliar, como intermediario entre la producción a ser un importante mecanismo de creación valor (Caro, 2014).

\section{Publicidad y derechos del consumidor}

La publicidad es un medio para transmitir información cuyo fin es dirigir la demanda hacia la empresa con el objetivo de incrementar las ventas, en este sentido resulta evidente que la empresa hará una selección de la información contenida en la publicidad acorde con el objetivo antes descrito. La capacidad de manipulación se manifiesta principalmente cuando la publicidad es engañosa tanto por el exceso como por defecto; por exceso cuando se incurre en exageraciones o falsedades sobre determinadas propiedades del producto o servicio; por defecto, si brinda información insuficiente y/o retacea información necesaria relativa a los atributos del bien. No debe perderse de vista que en cualquiera de los dos casos la publicidad puede inducir a las personas a las que se dirige a cometer errores en la decisión, ocasionar algún perjuicio y conducir a asignaciones socialmente ineficientes (Castellano \& Chaz, 2016).

\section{Bajo este precepto, la publicidad debe reunir tres requisitos fundamentales:}

Que sean veraces, es decir que los datos que contengan sean verdaderos, fidedignos correspondientes a las condiciones reales de los bienes o servicios anunciados

Que sean comprobables: lo que se traduce en que las características de los productos y servicios anunciados deben ser susceptibles a verificación y confirmación.

Que sean precisos: donde la información y la publicidad deberán estar exentas de textos, diálogos, sonidos, imágenes y demás descripciones que induzcan o puedan inducir a error o confusión, por su inexactitud. (Ovalle, 2000).

Por lo tanto, lo relevante es que los mensajes publicitarios cuenten con validez y brinden eficacia jurídica a los derechos del consumidor, aspecto que también dependerá de la clase de publicidad que se emplee (Escobar, 2018). 


\section{Metodología}

\section{Enfoque cuali-cuantitativo}

La investigación cualitativa es aquella que produce información descriptiva a partir de conductas observables (Quecedo \& Castaño, 2002). La investigación cuantitativa se enfoca hacia la búsqueda de resultados a base del manejo numérico y la estadística (Melero, 2011).

La presente investigación tuvo un enfoque mixto puesto que se utilizó el enfoque cuantitativo para el manejo riguroso de la información numérica desprendida de los instrumentos de recolección y el enfoque cualitativo empleado para la descripción completa y detallada de las variables.

\section{Tipo Descriptivo}

La investigación descriptiva detalla situaciones o eventos y busca especificar las propiedades del problema de estudio tal cual se presenta sin existir manipulación de las variables (Acosta, 2017). La investigación fue de tipo descriptiva en virtud de que se procedió a caracterizar las variables de estudio sin inferir en ellas de ningún modo y de esta manera obtener información real sobre la caracterización de la publicidad y su influencia en el consumidor del sector comercial de la Provincia de Tungurahua.

\section{Correlacional}

La Investigación correlacional pretende determinar la correlación entre las dos variables, misma que no implica causalidad y las variaciones se determinan estadísticamente (Acosta, 2017). El presente estudio se requirió de la investigación correlacional para establecer el nivel de dependencia existente entre las variables propuestas y con esto determinar si la publicidad influye en el consumidor del sector comercial tungurahuense.

\section{Métodos teóricos}

"Método que permite descubrir en el objeto investigativo las relaciones esenciales y cualidades fundamentales no detectables de modo sensoperceptual” (Martínez \& Rodríguez, 2018, p.4). 


\section{Entre los métodos teóricos empleados en la presente investigación se mencionan:}

Análisis-síntesis: método que permitió conocer de manera profunda la influencia de la publicidad y su influencia en el consumidor del sector comercial tungurahuense y de este modo simplificar su descripción y descubrir relaciones para la construcción de nuevos conocimientos.

Inducción -deducción: método empleado para la elaboración de la fundamentación teórica, análisis y revisión de resultados del estudio.

Histórico-lógico: método con el que se efectuó una revisión de la evolución del problema de estudio a través del tiempo, para analizar la trayectoria de la publicidad y su influencia en el consumidor del sector comercial de la provincia de Tungurahua examinando sus características, ventajas y dificultades.

\section{Métodos empíricos}

"Los métodos empíricos posibilitan revelar relaciones esenciales y las características fundamentales del objeto de estudio que posibles con detección sensoperceptual mediante métodos prácticos” (Martínez \& Rodríguez, 2018, p.4).

\section{Entre los métodos empíricos utilizados en el desarrollo investigativo están:}

Observación científica: método utilizado para recabar información relevante sobre la publicidad y su influencia en el consumidor, pero que no es proporcionada de manera directa por los informantes.

Encuesta: técnica que mediante un cuestionario estructurado fue aplicado a la población económicamente activa de la provincia de Tungurahua para obtener datos sobre aspectos relacionados con la publicidad y su influencia en el consumidor del sector comercial tungurahuense.

\section{Población y muestra}

Como población de estudio se considera al segmento económicamente activo de la provincia de Tungurahua que tras el cálculo de la tasa de crecimiento económico hacia 2018 es de 274.135 personas. Pero tomando en consideración que la población es demasiado extensa se procede al cálculo muestra: 


$$
n=\frac{Z^{2} P Q N}{Z^{2} P Q+N e^{2}}
$$

n: Tamaño de la muestra.

Z: Nivel de confianza 1.96

P: Probabilidad a favor de ocurrencia del fenómeno 50\%.

Q: Probabilidad en contra de ocurrencia del fenómeno 50\%.

N: Población de universo.

e: Nivel de error $5 \%$.

$$
\begin{gathered}
n=\frac{1.962 * 0.50 * 0.50 * 54510}{1.96^{2} * 0.50 * 0.50+54510 * 0.05^{2}} \\
n=\frac{52351.404}{0.9604+136.275} \\
n=\frac{52351.404}{137.2354} \\
n=384
\end{gathered}
$$

Para la aplicación de las 384 encuestas en los 9 cantones de la provincia de Tungurahua se realizó un cálculo proporcional, de este modo el instrumento de recolección se aplicó del siguiente modo:

Tabla 1 Distribución de encuestas por cantones

\begin{tabular}{|c|c|c|}
\hline CANTON & $\%$ PEA & \# ENCUESTAS \\
\hline Ambato & $65.8 \%$ & 253 \\
\hline Baños & $4.6 \%$ & 18 \\
\hline Cevallos & $1.5 \%$ & 6 \\
\hline Mocha & $1.2 \%$ & 5 \\
\hline Patate & $2.8 \%$ & 11 \\
\hline Pelileo & $11.4 \%$ & 44 \\
\hline Pillaro & $7.2 \%$ & 28 \\
\hline Quero & $3.9 \%$ & 15 \\
\hline Tisaleo & $1.3 \%$ & 5 \\
\hline Total & $100 \%$ & 384 \\
\hline
\end{tabular}

Elaborado por: Investigadora 


\section{Resultados y Discusión}

De las encuestas aplicadas a la población económicamente activa de Tungurahua, sobre aspectos de publicidad, influencia en el consumidor, se obtuvo la siguiente información:

Tabla 2 Resultados de las encuestas

\begin{tabular}{|c|c|c|}
\hline Interrogante & Indicadores & Resultados \\
\hline \multirow{4}{*}{$\begin{array}{l}\text { ¿Considera relevante la publicidad que presentan } \\
\text { las empresas del sector comercial? }\end{array}$} & Siempre & $48,9 \%$ \\
\hline & Casi siempre & $39,8 \%$ \\
\hline & Algunas veces & $9,3 \%$ \\
\hline & Casi nunca & $1,8 \%$ \\
\hline \multirow{4}{*}{$\begin{array}{l}\text { ¿Considera usted que las empresas del sector } \\
\text { comercial son creativas e innovan constantemente } \\
\text { la publicidad? }\end{array}$} & Siempre & $27,3 \%$ \\
\hline & Casi siempre & $44,2 \%$ \\
\hline & Algunas veces & $25,2 \%$ \\
\hline & Casi nunca & $3,1 \%$ \\
\hline \multirow{4}{*}{$\begin{array}{l}\text { ¿Considera usted que la publicidad es necesaria } \\
\text { para incrementar las ventas en el sector comercial? }\end{array}$} & Siempre & $47,4 \%$ \\
\hline & Casi siempre & $37,5 \%$ \\
\hline & Algunas veces & $13,2 \%$ \\
\hline & Casi nunca & $1,8 \%$ \\
\hline \multirow{4}{*}{$\begin{array}{l}\text { ¿Por qué medio publicitario usted se informa sobre } \\
\text { productos o servicios? }\end{array}$} & Radio & $31,5 \%$ \\
\hline & Televisión & $47,1 \%$ \\
\hline & Periódicos & $11,7 \%$ \\
\hline & $\begin{array}{l}\text { Publicidad exterior e } \\
\text { interior }\end{array}$ & $9,6 \%$ \\
\hline \multirow{4}{*}{$\begin{array}{l}\text { ¿Por qué redes sociales usted observa mayor } \\
\text { publicidad? }\end{array}$} & Facebook & $69,2 \%$ \\
\hline & Twitter & $16,4 \%$ \\
\hline & Instagram & $5,9 \%$ \\
\hline & YouTube & $8,3 \%$ \\
\hline \multirow{5}{*}{$\begin{array}{l}\text { ¿Qué tipo de mensaje publicitario considera que } \\
\text { debe utilizar el sector comercial? }\end{array}$} & Sorpresivo & $12,7 \%$ \\
\hline & Humorístico & $30,7 \%$ \\
\hline & Testimonial & $17,7 \%$ \\
\hline & Demostrativo & $18,4 \%$ \\
\hline & Informativo & $20,3 \%$ \\
\hline \multirow{5}{*}{$\begin{array}{l}\text { ¿Cree usted que el mensaje publicitario que emiten } \\
\text { las empresas es claro y real? }\end{array}$} & Siempre & $20,0 \%$ \\
\hline & Casi siempre & $41,4 \%$ \\
\hline & Algunas veces & $31,2 \%$ \\
\hline & Casi nunca & $5,9 \%$ \\
\hline & Nunca & $1,3 \%$ \\
\hline \multirow{5}{*}{$\begin{array}{l}\text { ¿Al momento de realizar una compra cuáles son } \\
\text { sus motivaciones? }\end{array}$} & Necesidad & $54,4 \%$ \\
\hline & Hábito & $17,7 \%$ \\
\hline & Impulso/gusto & $14,3 \%$ \\
\hline & Conveniencia & $7,8 \%$ \\
\hline & Prestigio/reconocimiento & $5,7 \%$ \\
\hline Importancia del precio en la decisión de compra & Muy importante & $29,6 \%$ \\
\hline
\end{tabular}


Caracterización de la gerencia publicitaria y su influencia en el consumidor del sector comercial de la Provincia de Tungurahua

\begin{tabular}{|c|c|c|}
\hline Importancia de la calidad en la decisión de compra & Muy importante & $28,1 \%$ \\
\hline Importancia del stock en la decisión de compra & Poco importante & $24,2 \%$ \\
\hline Importancia de la marca en la decisión de compra & Posición neutral & $29,4 \%$ \\
\hline $\begin{array}{l}\text { Importancia de promociones en la decisión de } \\
\text { compra }\end{array}$ & Muy importante & $24,4 \%$ \\
\hline \multirow{5}{*}{$\begin{array}{l}\text { ¿Considera que la publicidad influye en la decisión } \\
\text { de compra }\end{array}$} & Siempre & $32,8 \%$ \\
\hline & Casi siempre & $46,3 \%$ \\
\hline & Algunas veces & $16,4 \%$ \\
\hline & Casi nunca & $2,0 \%$ \\
\hline & Nunca & 2,0 \\
\hline \multirow{5}{*}{$\begin{array}{l}\text { ¿Con respecto a la procedencia de los productos, } \\
\text { ¿cuáles son de su preferencia? }\end{array}$} & Locales & $24,4 \%$ \\
\hline & Regionales & $30,7 \%$ \\
\hline & Nacionales & $28,9 \%$ \\
\hline & Extranjeros & $15,6 \%$ \\
\hline & Otros & $0,2 \%$ \\
\hline \multirow{4}{*}{$\begin{array}{l}\text { ¿Considera que es necesario diseñar estrategias de } \\
\text { publicidad para el sector comercial de la provincia } \\
\text { de Tungurahua? }\end{array}$} & Siempre & $45,3 \%$ \\
\hline & Casi siempre & $40,8 \%$ \\
\hline & Algunas veces & $12,2 \%$ \\
\hline & Casi nunca & $1,5 \%$ \\
\hline
\end{tabular}

Elaborado por: Investigadora

Los resultados de la presente investigación ponen en evidencia que la mayor parte del sector comercial asume que la publicidad es fundamental para el incremento de las ventas, por lo que optan por dar a conocer sus productos y/o servicios por medios publicitarios tradicionales como la televisión, pero también se apoyan en medios tecnológicos como las redes sociales, principalmente Facebook. Respecto a la manera en que la publicidad influye en el consumidor se determinó que la principal motivación de compra es la necesidad, pero además que se considera como factores importantes a la calidad, precio y promociones.

Por lo tanto, los resultados denotan que la publicidad se relaciona con el incremento de las ventas en el sector comercial al ser un factor determinante e influyente en el consumidor. Información que se sustenta en lo expuesto por Camino (2014) quien destaca que la publicidad es un factor clave para cualquier empresa, al permitir ofrecer información acerca de la oferta de productos a los clientes potenciales, además de influenciar en los consumidores cuando la competencia ofrece productos similares ya que una publicidad es atrayente, hace que las masas consuman más y consecuentemente el producto se posicione en el mercado. 
A lo expuesto, Traillanca (2012) añade que existe una relación muy estrecha entre la publicidad y el consumidor ya que la producción masiva de bienes de consumo exige también de una venta masiva de esos productos y esta venta sólo puede llevarse a cabo con la ayuda de la publicidad, puesto que la publicidad aumenta la demanda de determinados productos por parte de los consumidores al despertar en éstos la necesidad de adquirirlos. (p.2). Así, se asume que la publicidad tiene gran utilidad respecto a las ventas ya que cuanto mayor sea la persuasión comercial, mayor será la intención de compra y cuanto mayor sea la publicidad, mayor será la valoración de las marcas.

\section{Propuesta de solución}

En base a los resultados obtenidos y a las necesidades del sector se planteó una estrategia de publicidad a base de redes sociales para mejorar las ventas del sector comercial de la Provincia de Tungurahua, en virtud que las redes sociales son espacios virtuales que pueden ser utilizados para desarrollar proyectos, integrar comunidades, las redes sociales están siendo empleadas como un canal de influencia y comunicación alterna para cualquier tipo de empresa y se ha consolidado como un poderoso medio para atender y gestionar clientes mucho más efectivos que cualquier tipo de medio dedicado a promocionar productos o servicios. Estas estrategias se esquematizan a continuación:

Tabla 3 Propuesta de solución

\section{Definición de metas}

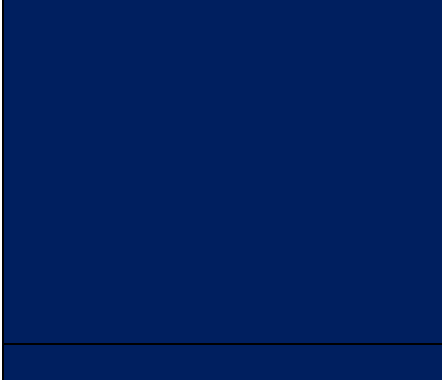

Asegurar el respaldo de socios y colaboradores
- Deben ser específicas y adaptadas a la realidad de la empresa

- Deben adaptarse a la visión y misión de la marca.

- Deben ser realizables con los recursos de la empresa

- Deben ser medibles

- Deben plantearse a largo plazo

- Informar sobre los beneficios del uso de redes sociales como medio publicitario

- Elegir redes sociales adecuadas

- Asignar y programar el presupuesto

- Seleccionar el personal para el manejo de redes sociales

- Todo el grupo empresarial debe enfocarse al crecimiento y posicionamiento de la marca en redes sociales 


\begin{tabular}{|l|l|}
\hline Ejecución & Creación de páginas: personalización y \\
& captación de clientes, seguimiento. \\
& - Manejo de páginas: publicaciones fijas y \\
& ocasionales. \\
& - Mantenimiento de páginas: actualizaciones, \\
& nueva información. \\
\hline Estrategias & - Imagen de marca \\
& - Ventas online \\
& - Estudios de mercado \\
& - Fidelización de clientes \\
& - Captación de leads \\
\hline Evaluación & Analizar indicadores de resultados \\
\hline
\end{tabular}

Elaborado por: Investigadora

\section{Conclusiones}

En la presente investigación se encontró que la publicidad es fundamental para el incremento de las ventas en el sector comercial al ser un factor determinante e influyente en el consumidor ya que una publicidad hace que las masas consuman más y consecuentemente el producto se posicione en el mercado.

Se identificó que el tipo de publicidad tradicional más utilizado e influyente con el consumidor en el sector comercial de la Provincia de Tungurahua es la televisión al ser de dominio público. Pero demás, se ha generalizado la publicidad en redes sociales debido a la era digital, en la que la mayoría de las personas utiliza un aparato tecnológico como herramienta de primera mano para la adquisición de productos y/o servicios.

Según los estudios pertinentes elaborados, en más de un 95\% de los casos, se puede afirmar que la motivación de compra está influenciada por la publicidad de cualquier tipo que esta fuera, así como la persuasión y la valoración de las marcas.

Finalmente, se diseñó una guía de publicidad a base de redes sociales en la que se brinda recomendaciones sobre creación, manejo, mantenimiento de estas plataformas y estrategias para mejorar las ventas del sector comercial de la Provincia de Tungurahua, pues actualmente las redes 
sociales se han consolidado como un poderoso medio para atender y gestionar clientes mucho más efectivos que cualquier otro tipo de medio.

\section{Referencias Bibliográficas}

Acosta, S. (2017). Fundamentos de Metodología de la Investigación: tipos y diseños de la Investigación. México: Universidad Autónoma del Estado de Hidalgo

Akrani, G. (2013). Importance of advertising-why advertising is important? Kalyan City Life.

Bárcena, L. (2014). Propaganda y publicidad. México: Universidad Autónoma del Estado de Hidalgo.

Cadena, M. (2014). El marketing con causa como estrategia de marca para las empresas Ecuatorianas. Quito: Universidad San Francisco de Quito.

Camino, J. (2014). Estrategias de publicidad y su impacto en las ventas de la Empresa Repremarva de la ciudad de Ambato, durante el año 2012. Ambato: Universidad Técnica de Ambato.

Cañar, T., \& Guzmán, F. (2014). Investigación sobre la percepción del mercado respecto a la publicidad ATL en los medios de comunicación en la ciudad de Cuenca. Cuenca: UPS

Caro, A. (2014). Comprender la Publicidad para Transformar la Sociedad. Revista Scielo.

Corbacho, J. (2009). La publicidad a nivel internacional: Estrategias, condiciones, límites. España: Universidad de Vigo.

De Durán, A. (2014). Planificación estratégica publicitaria. Universidad Rey Juan Carlos.

Esteinou, J. (2014). La publicidad y la transformación cultural del país. Revista Scielo.

Guera, M. (2006). La protección al consumidor por publicidad engañosa en el Ecuador y sus limitaciones legales de acuerdo a la legislación vigente. Quito: Universidad Andina Simón Bolívar.

Herrera, B. (2013). La constitucionalización de los derechos del consumidor en Colombia: un análisis desde los derechos sociales fundamentales. Revista Scielo. 
Martínez, R., \& Rodríguez, E. (2018). Manual de Metodología de la Investigación Científica. Factory.

Melero, N. (2011). El paradigma crítico y los aportes de la investigación acción participativa en la transformación de la realidad social: un análisis desde las ciencias sociales. España: Universidad de Sevilla.

Molina, G. (2008). El procedimiento para la reparación de los derechos del consumidor en el Ecuador, análisis de 3 casos, propuesta de reforma. Quito: Universidad Andina Simón Bolívar.

Novo, J (2016). “Publicidad, persuasión y comportamiento del consumidor”. Universidad de Coruña

Núñez, Olarte \& Reinares, (2014). Influencia de la publicidad en las tendencias sociales: Una aproximación exploratoria al mercado publicitario Español. Universidad de la Rioja.

Oller, M. (2016). Tendencias y retos del marketing en Ecuador 2015. Quito: q.

Quecedo, R., \& Castaño, C. (2002). Introducción a la metodología de investigación cualitativa. Revista de Psicodidáctica, 7.

Terkan, R. (2014). Importance of Creative Advertising and Marketing According to University

Traillanca, D. (2012). Impacto de la publicidad comercial de las tiendas Retail en el comportamiento de compra de los consumidores. Puerto Mont: Universidad Austral de Chile.

Vinatea, R. (2013). El derecho del consumidor como nuevo paradigma del derecho en una economía social de mercado. Corte Superior de Lima.

Yánez, S. (2015). La publicidad en la comunicación organizacional. Quito: Universidad Central del Ecuador. 